\title{
Medo de cair em idosos e modelos de intervenção psicoterápica
}

\author{
Fear of falling in the elderly and psycotherapic intervention models
}

\section{Miedo a caer en los adultos mayores y modelos de intervención psicoterápica}

\author{
Wallace H. dos Santos*, Karen O. Santos
}

\begin{abstract}
Resumo
O objetivo do presente estudo é fazer uma revisão bibliográfica dos modelos de intervenção psicoterápica utilizados em idosos que apresentam "medo de cair". A pesquisa bibliográfica foi realizada nas bases MEDLINE, LILACS e SciELO, e foram acessados dez estudos com diferentes desenhos e intervenções, dos quais dois eram artigos de revisão sobre o medo de cair em idosos. Dentre os oito artigos que apresentaram modelos de intervenção, sete foram pautados na terapia cognitivo-comportamental (TCC) e um não deixou clara a linha de condução, sendo que os estudos que apresentaram melhor resultado na prática clínica foram os de intervenção multidisciplinar. Os dois estudos encontrados se valeram da intervenção da Fisioterapia e da Psicologia para avaliação e tratamento do medo de cair em idosos. Apesar de se apresentar como uma questão de saúde pública, existe uma escassez de trabalhos sobre modelos para intervenção e recuperação dos possíveis impactos psíquicos que influenciam a rotina de idosos com esse medo. O principal achado deste trabalho foi a caracterização do medo de cair como uma fobia, logo ligada a um transtorno de ansiedade, o que ajuda a nortear o tratamento, prognóstico e plano de ação dos profissionais envolvidos. Conclui-se que a revisão de modelos existentes para o medo de cair precisa ser expandida em âmbito mundial e que se deve pautar as pesquisas conforme os modelos com os melhores resultados. Esse amadurecimento e a estruturação no campo do conhecimento do psíquico dos idosos podem vir através de uma maior participação dos psicólogos em pesquisas, adaptações e ensaios de modelos para o medo de cair, assim como para outros quadros específicos e para a avaliação inicial, com a população idosa.
\end{abstract}

Descritores: Psicoterapia; Medo; Idoso; Acidentes por quedas.

\begin{abstract}
The goal of this study is to review the psychotherapeutic intervention models applied to elderly who have "fear of falling" and to identify the results. The literature search was performed on MEDLINE, LILACS and SciELO and ten studies with different designs and interventions were accessed, including two review articles about the fear of falling in the elderly. Among the eight articles that showed intervention models, seven were based on the cognitive-behavioral therapy (CBT) and one was not clear about its conducting line. The studies that showed better results in clinical practice used the multidisciplinary intervention. These two multidisciplinary studies used psychology and physiotherapy for assessment and treatment of the fear of falling in the elderly, with good effect on health after a six-month follow-up. Although it presents itself as a public health issue, there is a lack of studies on models for intervention and recovery of possible impacts that influence routine from elderly with fear of falling. The main finding of this study was the characterization of the fear of falling as a phobia, soon connected to an anxiety disorder, which helps to guide treatment, prognosis and the plan of action of the professionals involved. It was concluded that a review of existing models for the fear of falling must be expanded worldwide and that research should be based on the models with the best results. This maturing and structuring of knowledge in the field of elderly psyche can come through greater involvement of psychologists in the research, adjustments and tests of models for the fear of falling, as well as for other specific conditions and for the initial evaluation, within the elderly population.
\end{abstract}

Keywords: Psychotherapy; Fear; Aged; Accidental falls. 


\section{Resumen}

El objetivo del presente estudio es hacer una revisión bibliográfica de los modelos de intervención psicoterápica utilizados en adultos mayores que presentan "miedo a caer". La investigación bibliográfica fue realizada en la base del MEDLINE, LILACS e SciELO y se accedieron a diez estudios con diferentes diseños e intervenciones, de los cuales dos eran artículos de revisión sobre el miedo a caer en adultos mayores. De los ocho artículos que presentaron modelos de intervención, siete fueron listados en la terapia cognitivo-comportamental (TCC) y uno no dejó clara la línea de dirección, verificándose que los estudios que presentaron mejor resultado en la práctica clínica fueron los de intervención multidisciplinar. Los dos estudios encontrados se valieron de la intervención de la Fisioterapia y de la Psicología para la evaluación y tratamiento del miedo a caer en adultos mayores. A pesar de presentarse como una cuestión de salud pública, existe una escasez de trabajos sobre los modelos de intervención y de recuperación de los posibles impactos psíquicos que influencian la rutina de los adultos mayores con ese miedo. El principal hallazgo de este trabajo fue la caracterización del miedo a caer como fobia, por lo tanto relacionado con un trastorno de ansiedad, lo que ayuda a orientar el tratamiento, pronóstico y plan de acción de los profesionales implicados. Se concluye que la revisión de modelos existentes sobre el miedo a caer necesita ser ampliada en el ámbito mundial y que se deben direccionar las investigaciones sobre los modelos con los mejores resultados. Esta maduración y estructuración en el campo de conocimiento del psíquico de los adultos mayores puede darse a través de una mayor participación de los psicólogos en investigaciones, adaptaciones y ensayos de modelos sobre el miedo a caer, así como para otros cuadros específicos y para la valoración inicial, con la población adulta mayor.

Palabras clave: Psicoterapia; Miedo; Adulto mayor; Accidentes por caídas.

\section{Introdução}

O objetivo do presente estudo é rever os modelos de intervenção psicoterápica utilizados em idosos que apresentam "medo de cair" e identificar os resultados encontrados. Pode-se definir queda como "um evento não intencional que tem como resultado a mudança de posição do indivíduo para um nível mais baixo em relação a sua posição inicial”.' Por trazer, comumente, consequências negativas à saúde dos idosos, observamos este evento como uma das causas externas mais importantes nesta população, descrita na Classificação Internacional de Doenças (CID-10). ${ }^{2}$

As quedas em idosos constituem um problema de saúde pública com potencial de causar dependência funcional, seja por sequelas físicas ou pelo prejuízo psicológico aos indivíduos e à sua família. ${ }^{3}$ As causas podem ocorrer por fatores extrínsecos, como circunstâncias sociais e o ambiente, ou por fatores intrínsecos, decorrentes de alterações fisiológicas relacionadas ao enveIhecimento. ${ }^{4} \mathrm{O}$ caráter multifatorial e heterogêneo nos leva a considerar este evento como uma das principais síndromes geriátricas, ${ }^{3,4}$ com desfechos importantes, como imobilidade e incontinência, ao que apenas 50\% dos idosos com 75 anos ou mais estarão vivos após um ano da hospitalização por queda. ${ }^{5}$

Segundo dados do Sistema de Informação Mé-
dica/Ministério da Saúde, foram registrados cerca de 54.730 óbitos em decorrência de quedas entre os anos de 1979 e 1995 no Brasil, sendo 52\% das ocorrências com idosos. De acordo com os dados do Ministério da Saúde/Sistema de Informação Hospitalar, a taxa de mortalidade hospitalar por queda foi de $2,58 \%$ em fevereiro de 2000 , sendo a maior prevalência na região Sudeste. ${ }^{4}$

Já em 2005, o percentual de hospitalizações por queda entre os idosos atingiu 3,7\% de todas as internações de idosos no estado do Rio de Janeiro. ${ }^{6}$ Em 2007, segundo o banco de dados do Sistema Único de Saúde (DATASUS), o Rio apresentou 1.947 internações de idosos de 60 a 69 anos por quedas, correspondendo a $57 \%$ dos registros, e 3.755 na faixa etária de 70 anos ou mais, que representam mais de $68 \%$ das internações.?

Em estudo realizado na região metropolitana de São Paulo observou-se a prevalência de quedas em $30 \%$ dos idosos. ${ }^{8}$ Fabrício e colaboradores ${ }^{4}$ apresentam o medo de cair como a segunda consequência mais prevalente, cerca de $40 \%$, em seu estudo, atrás apenas de fraturas. Podemos observar, muitas vezes, a restrição de atividades físicas, modificações nas atividades de vida diária (AVDs) e nas atividades instrumentais de vida diária (AIVDs). ${ }^{6} \mathrm{O}$ relato de medo de cair aparece também em quadros específicos ligados ao equilíbrio e à marcha, como no caso de idosos com vestibulopatia crônica, em que o medo de queda 
e a tendência a quedas são referidos pela maioria dos sujeitos. ${ }^{9}$

No cenário internacional, Tinetti ${ }^{10}$ afirma que mais de um terço das pessoas com 65 anos ou mais cai todo ano e em metade dos casos as quedas são recorrentes. Aproximadamente uma em cada dez quedas resulta em ferimento sério, usualmente dos tecidos moles, podendo causar também lesões graves, tais como fratura de quadril ou traumatismo cranioencefálico.

As quedas são causas de aproximadamente $10 \%$ das emergências hospitalares e $6 \%$ das hospitalizações urgentes, estando associadas à restrição de mobilidade e declínio na capacidade de realizar atividades cotidianas como vestir-se, banhar-se, fazer compras ou administrar o lar, e ao risco crescente de institucionalização. ${ }^{3}$

O Centro Latino Americano de Estudos de Violência e Saúde (CLAVES), da Fundação Oswaldo Cruz, encontrou a influência das quedas sobre a qualidade de vida dos idosos estudados, em todos os domínios do WHOQOL-Bref. ${ }^{6}$ Houve redução nas médias do grupo que caiu no último ano em relação aos que não caíram, assim como a diferença mais significativa no domínio psicológico, com $88,5 \%$ dos idosos indicando que passaram a sentir medo de cair.

Os achados sobre as alterações psíquicas nos conduziram a uma revisão sobre o medo de cair e dos modelos psicoterápicos de intervenção, tendo como ponto de partida a Falls Efficacy Scale (FES) e as discussões de Tinetti e colaboradores, ${ }^{10-13}$ que chamam a atenção pelo pioneirismo no campo e por tratar sob um olhar basicamente fisiopatológico, através da saúde pública e da epidemiologia, de um evento que tem associação direta com causas e consequências psicológicas e psiquiátricas.

Neste contexto, o presente artigo se propõe a estudar o que o campo da psicologia tem produzido a esse respeito, ou seja, quais as intervenções específicas voltadas à população idosa quanto ao medo de cair e suas repercussões na saúde do indivíduo, e tem como foco uma descrição detalhada de consequências psicológicas e psiquiátricas. ${ }^{5}$ Nesta, os autores enumeram dez fatores importantes como consequências das quedas e destacam o impacto emocional, o medo de cair e a síndrome pós-queda como pontos relevantes, estando o medo presente em idosos que caíram e nos que não sofreram quedas acidentais. ${ }^{10,14}$
Verificamos uma alta frequência do medo de cair em idosos nos dados dos estudos encontrados. Alguns resultados apontam que de $20 \%$ a $60 \%$ dos idosos referem o medo de cair. ${ }^{15-18} \mathrm{Em}$ idosos com 80 anos ou mais, Manckoundia e colaboradores identificaram a ocorrência deste temor descrita por cerca de $50 \%$ deste grupo..$^{19}$ Os estudos citados avaliaram os idosos nas comunidades em que vivem, com variação em graus de leve a grave.

Este quadro tem chamado a atenção por sua incidência, como observamos, e também por seus reflexos na saúde global do idoso, mais especificamente nas atividades de vida diária ou na chamada idade funcional. ${ }^{20}$ As restrições geradas pelo medo de cair têm como consequência a evitação de algumas atividades que antes eram desempenhadas sem problemas. O medo após a queda pode trazer consigo não somente o medo de novas quedas, mas também de se machucar, ser hospitalizado, sofrer imobilizações, ter declínio de saúde, tornar-se dependente de outras pessoas para o autocuidado ou para realizar atividades da vida diária, ou seja, medo das consequências inerentes à queda. ${ }^{4}$

Em um artigo de revisão sobre o medo de cair, Gail e colaboradores apontam a descrição de síndrome pós-queda e ptofobia como condições associadas ao medo de cair e a quedas. ${ }^{3}$ A síndrome pós-queda foi definida como o resultado final da combinação da instabilidade postural não tratada com a queda propriamente dita, a qual resulta em danos à saúde física e mental do indivíduo idoso. O termo ptofobia foi usado para designar a reação fóbica para se manter em pé e andar, relacionada ao medo de cair: mesmo não havendo nenhuma anormalidade neurológica ou ortopédica, o indivíduo não consegue andar sem se apoiar. ${ }^{3}$

Ambos os conceitos, ptofobia e síndrome pós-queda, apresentam em comum o medo de cair, discordam na obrigatoriedade de um evento de queda e indicam fatores psicológicos como desfecho comum. Utilizaremos o foco em componentes ansiosos ou sintomas depressivos, dentre outros, sem a discriminação do quadro ou nomenclatura específica, para verificar quais intervenções psicoterápicas foram propostas. Para medo de cair usaremos: uma falta de autoconfiança, que pode conduzir a restrição nas $\mathrm{AVDs}^{19} \mathrm{e}$ a forma abreviada do inglês FOF (fear of falling).

Há uma escassez na literatura pesquisada sobre os modelos de intervenção psicoterápica 
para idosos com medo de cair. Não foram encontradas ocorrências na busca por artigos no Brasil, no banco de dados LILACS e base da SciELO com os descritores psicoterapia, idoso e medo de cair.

Apesar das consequências do medo de cair trazerem desafios no âmbito psicológico e psiquiátrico, com repercussões em diferentes domínios (como, por exemplo, na evitação de atividades), com reflexos no humor, em sintomas ansiosos, na autoeficácia percebida, na qualidade de vida, dentre outros fatores de interferência mútua sobre a saúde global do indivíduo, o problema não tem sido suficientemente estudado entre nós. Com base no exposto, optamos pela condução deste estudo através dos modelos disponíveis na literatura de intervenção psicoterápica para idosos com medo de cair e pela avaliação de seus resultados para, então, discutirmos o uso dessas estratégias.

\section{Metodologia}

Como não encontramos resultados para a busca por psicoterapia, medo de cair e idoso no banco de dados LILACS nem na SciELO, nesta terceira fonte (http://bireme.br) a busca foi restrita à coleção do Brasil. No MEDLINE, foram encontrados dez estudos da combinação dos três temas.

A pesquisa bibliográfica foi realizada na base de dados do MEDLINE, sem limites quanto ao período inicial da busca pela data de publicação, e se estendeu até setembro de 2009. Utilizou-se o Medical Headings (MeSH) para a seleção dos termos e para a busca por descritores. Os critérios de inclusão foram: idade (ages) 65+; estudos com humanos (humans); e língua (language) inglês e português. Não houve delimitação quanto à abordagem teórico-metodológica.

Numa visão geral do MeSH e das palavras usadas para busca eletrônica no banco de dados, encontramos dez estudos para a pesquisa combinada de idoso, psicoterapia e medo de cair, dentre os quais dois artigos de revisão sobre o medo de cair em idosos. ${ }^{3,21}$

\section{Resultados}

Dos oito estudos que completaram os critérios de inclusão, encontramos quatro estudos engajados na tradução e adaptação do programa A Matter of Balance, um modelo norte americano, para a população holandesa, ${ }^{15-17,22}$ denominado AMB-NL. Estes estudos apresentam diferentes fases do mesmo projeto, desenvolvido por um grupo de profissionais - van Haastregt, Zijlstra e van Eijk -, todos pautados na intervenção pela terapia cognitivo-comportamental (TCC).

Além desses, há um estudo comparativo entre dois grupos distintos, um com atividade física e o outro grupo de intervenção educacional para identificação e redução dos riscos de quedas. ${ }^{18}$ Noutro estudo foi realizada a comparação entre diferentes variáveis mentais, significativas na literatura revisada, para a recuperação funcional após cirurgia de fratura de bacia em idosos, no qual o medo de cair manteve-se como um preditor mais importante do que a dor e a depressão, na avaliação de 187 sujeitos em quatro unidades ortopédicas de Manchester, no Reino Unido. ${ }^{23}$

A associação do medo de cair com estados mentais comuns da vida tardia, como depressão e ansiedade, foi verificada em percentuais diferentes nos 540 participantes do estudo: ${ }^{15}$ 21,7\% apresentavam sintomas ansiosos, $18,4 \%$ sintomas de depressão e 10,7\% a combinação dos dois. Estes dados foram coletados em duas zonas urbanas da Holanda. A severidade do medo de cair está associada à presença de sintomas depressivos, e a severidade da evitação de atividades, aos sintomas ansiosos. ${ }^{15}$

Os estudos que apresentaram melhor resultado na prática clínica foram os de intervenção multidisciplinar. Ambos se valeram da intervenção da Fisioterapia e da Psicologia para avaliação e tratamento do medo de cair em idosos e lançaram mão da TCC como modelo-guia das intervenções sobre este evento. ${ }^{19,24}$

Em Bhala, O'Donnell e Thoppil, ${ }^{24}$ cinco dos seis pacientes foram totalmente recuperados, com baixa da ansiedade e aumento da funcionalidade, após dez sessões conduzidas por um psicólogo com técnicas de dessensibilização, concomitante ao tratamento fisioterápico, e nos resultados foi ressaltada a importância do reforço, verbal e físico, pelo terapeuta e pelo meio social, para a recuperação dos pacientes.

Ganhos na redução do FOF por uma intervenção multidisciplinar, envolvendo fisioterapeuta e psicólogo, também foram encontrados para pacientes com quadro de síndrome de desadaptação psicomotora e histórico de quedas. ${ }^{19} \mathrm{Em}$ 
suma, encontramos oito artigos na literatura que apresentaram um modelo de intervenção com referência ao aspecto psicológico, assim como aos resultados de tais intervenções.

\section{Discussão}

Percebemos uma alta prevalência dos modelos de intervenção em TCC, em que sete dos oito artigos traziam esse viés. A TCC vem se destacando pelo seu foco no tratamento, com sessões estruturadas e por sua prática atrelada aos modelos médicos de diagnósticos existentes.

Esta abordagem psicoterapêutica possui um viés pedagógico importante para o tratamento de idosos com medo de cair, porém ainda se faz necessária uma adaptação por parte da TCC, e da psicologia como um todo, à prática específica e voltada para a população idosa.

$\mathrm{O}$ que se percebe pelos resultados encontrados nessa busca por modelos de intervenção é uma escassez de produção técnico-científica e de técnicas formalizadas para um uso mais amplo na sociedade. Apesar de se tratar de um problema de saúde pública, com as taxas de prevalência observadas anteriormente, os profissionais de psicologia com enfoque na gerontologia parecem não estar atentos para a urgência em pesquisas e produção de modelos adequados para o tratamento de FOF em idosos.

Esta escassez abre brechas, como vemos no estudo de Brouwer e colaboradores,${ }^{18}$ no qual não fica claro que tipo de profissional está à frente da condução do grupo educacional e se esta era realizada conforme algum modelo específico da psicologia ou psiquiatria para grupos. O que se apresenta é apenas o resultado mais favorável, na escala SF-36, no domínio da saúde mental em comparação com o grupo de atividade física.

Vale ressaltar que os estudos que apresentaram com clareza o papel do profissional de saúde mental e o modelo de intervenção bem delimitado para redução de FOF em idosos demonstraram também resultados favoráveis..$^{19,24}$ Estes achados corroboram a importância de um olhar especializado, no campo da saúde mental e da gerontologia, para o tratamento, recuperação, condução e elaboração de modelos para o medo de cair em idosos. Apesar da maior parte dos estudos envolverem avaliação, as intervenções multidisciplinares obtiveram melhores resultados do que outros estudos que compararam modelos.

Porém, não é possível concluir qual é o modelo mais adequado para o tratamento do medo de cair em idosos pela quantidade reduzida de estudos encontrados, assim como bons resultados de intervenções multidisciplinares no tratamento amplo e a avaliação global da pessoa idosa não são passíveis de generalização. Existem trabalhos desenvolvidos e em andamento nos ambulatórios da Universidade Aberta da Terceira Idade da UERJ (UnATI/UERJ) - Cuidado Integral à Pessoa Idosa (CIPI) e Núcleo de Atenção ao Idoso (NAl) que poderiam contribuir para o treinamento e ensaios de modelos multidisciplinares na área.

O presente estudo é importante para o levantamento da questão referente ao enfrentamento psicológico do medo de cair em pessoas idosas e de estratégias clínicas nos serviços de atenção especializados em geriatria e gerontologia. Os achados sobre os modelos psicoterápicos e seus resultados para o tratamento deixam clara a necessidade de inserção dessas questões no topo da ordem do dia, quer em termos de políticas públicas, quer em termos de saúde suplementar, assim como a exigência de que novas formas de intervenção sejam pensadas e colocadas em prática.

Apesar da escassez de literatura encontrada, foi possível indicar rumos e sugerir adaptações para a prática dentro dos serviços ambulatoriais e da clínica particular voltada para a terceira idade, com uma provocação teórica aos profissionais que pretendem se debruçar sobre o assunto. Assim, o principal achado foi a descrição do medo de cair como uma fobia, logo, um transtorno de ansiedade, o que ajuda a nortear os aspectos dos estudos e intervenção clínica.

Vale relembrar que a depressão também apareceu como um importante indicador clínico, quanto à severidade do FOF e os sintomas ansiosos ligados à evitação de atividades. Estes dois quadros clínicos, ansiedade e depressão, possuem uma grande taxa de comorbidade, aspecto relevante para traçar estratégias de pesquisa e tratamento.

A partir daí, o psicólogo deve auxiliar no direcionamento das demandas desse cliente, identificando os recursos que cada profissional de uma equipe multidisciplinar possui, assumindo, desse modo, o papel que the cabe neste contexto. Tal 
papel consiste em centralizar o cuidado emocional e psíquico desse paciente para se alcançar efetivamente resultados em uma situação limitante que é inerente à capacidade funcional, em outras palavras, mais relacionada à autonomia da pessoa idosa do que a seu grau de independência.

Com o aumento da produção científica e a adaptação de modelos consagrados para morbidades relacionados a fobias em outras populações etárias para uma visão especializada em idosos, como a inserção de um viés pedagógico nas intervenções e também de um olhar para a rede de suporte social e familiar, imagina-se que ficariam poucas lacunas ou arestas para futuras pesquisas sobre a prática clínica ligada ao medo de cair em idosos, visto que outros domínios, como o físico e funcional, já estão bem estruturados e descritos.

\section{Conclusão}

Concluiu-se que existem várias lacunas a serem preenchidas quanto a modelos de intervenção sobre o medo de cair em idosos e que estas podem ser um reflexo da escassez de produção dos psicólogos que trabalham com envelhecimento humano em temas e alterações específicas, mesmo com alto índice de prevalência do medo de cair em idosos na sociedade. Por vezes, é possível encontrar vasta produção ligada a alguns temas, como a depressão; contudo, apenas uma pequena minoria apresenta ou discute modelos de intervenção psicoterápica.

Aponta-se também a dificuldade numa ampla elaboração de conceitos, teoria ou modelos, visto que a quantidade de estudos relacionados ao tema ainda é escassa. Apesar de a prática clínica multiprofissional sugerir e apresentar bons resultados em alguns contextos ambulatoriais de atendimento à população idosa, é necessário que se avalie a aplicabilidade e capacidade de reproduzir procedimentos e intervenções em diferentes contextos, nos quais, por exemplo, as variáveis de profissionais qualificados, de recursos e de capacidade acesso podem ser limitadores da práxis e da pesquisa.

\section{Referências}

1. Moura RN, Driemeier M, Santos LM, Ramos LR. Quedas em idosos: fatores de risco associados. Gerontologia. 1999;7(2):15-21.
2. Organização Mundial da Saúde. CID-10. São Paulo; 2000. p. 1017-1019.

3. Gai J, Gomes L, Cárdenas CJ. Ptofobia: o medo de cair em pessoas idosas. Acta Med Port. 2009;22(1):83-8.

4. Fabrício SCC, Rodrigues RAP, Costa ML Jr. Causas e consequências de quedas de idosos atendidos em hospital público. Rev Saúde Pública. 2004;38(1):93-9.

5. Rocha FL, Cunha UGV. Aspectos psicológicos e psiquiátricos das quedas do idoso. Arq Bras Med. 1994;68(1):9-12.

6. Ribeiro AP, Souza ER, Atie S, Souza AC, Schilithz AO. A influência das quedas na qualidade de vida de idosos. Ciênc Saúde Colet. 2008;13(4):1265-73.

7. DATASUS. Indicadores de morbidade e fatores de risco. Brasília: Ministério da Saúde [acesso em 11/09/2011]. Disponível em: http://tabnet.datasus.gov. br/cgi/tabcgi.exe?idb2008/d14.def

8. Perracini MR, Ramos LR. Fatores associados a quedas em uma coorte de idosos residentes na comunidade. Rev Saúde Pública. 2002;6(36):709-16.

9. Ganança FF, Gazzola JM, Aratani MC, Perracini MR, Ganança MM. Circunstâncias e consequências de quedas em idosos com vestibulopatia crônica. Rev Bras Otorrinolaringol. 2006;72(3):388-93.

10. Tinetti ME, Richman D, Powell L. Falls efficacy as a measure of fear of falling. J Gerontol. 1990;45(6):P239-43.

11. Tinetti ME, Powell L. Fear of falling and low selfefficacy: a case of dependence in elderly persons. J Gerontol. 1993;48(Spec Issue):35-8.

12. Tinetti ME, Leon CFM, Doucette JT, Baker DI. Fear of falling and fall-related efficacy in relationship to functioning among community-living elders. J Gerontol. 1994;49(3):M140-7.

13. Tinetti ME. Clinical practice. Preventing falls in elderly persons. N Engl J Med. 2003;348(1):42-9.

14. Scheffer AC, Schuurmans MJ, van Dijk N, van der Hooft T, Rooji SE. Fear of falling: measurement strategy, prevalence, risk factors and consequences among older persons. Age Ageing. 2008;37(1):19-24.

15. van Haastregt JC, Zijlstra GA, van Rossum E, van Eijk JT, Kempen GI. Feelings of anxiety and symptoms of depression in community-living older persons who avoid activity for fear of falling. Am J Geriatr Psychiatry. 2008;16(3):186-93.

16. van Haastregt JC, Zijlstra GA, van Rossum E, Eijk JT, Witte LP, Kempen GI. Feasibility of a cognitive behavioural group intervention to reduce fear of falling and associated avoidance of activity in community-living older people: a process evaluation. BMC Health Serv Res. 2007;7:156. 
17. Zijlstra GA, Tennstedt SL, van Haastregt JC, van Eijk JT, Kempen GI. Reducing fear of falling and avoidance of activity in elderly persons: the development of a Dutch version of an American intervention. Patient Educ Couns. 2006;62(2):220-7.

18. Brouwer BJ, Walker C, Rydahl SJ, Culham EG. Reducing fear of falling in seniors through education and activity programs - a randomized trial. J Am Geriatr Soc. 2003;51(6):829-34.

19. Manckoundia P, Gerbault N, Mourey F, d'Athis P, Nourdin C, Monin MP, et al. Multidisciplinary management in geriatric day-hospital is beneficial for elderly fallers: a prospective study of 28 cases. Arch Gerontol Geriatr. 2007;44(1):61-70.

20. Veras R, Lourenço R (Eds.). Formação humana em Geriatria e Gerontologia. Rio de Janeiro: UnATI/ UERJ; 2006. 344 p.
21. Evitt CP, Quigley PA. Fear of falling in older adults: a guide to its prevalence, risk factors, and consequences. Rehabil Nurs. 2004;29(6):207-10.

22. Zijlstra G, van Haastregt JC, van Eijk JT, Kempen GIJM. Evaluating an intervention to reduce fear of falling and associated activity restriction in elderly persons: design of a randomised controlled trial. BMC Public Health. 2005;5:26.

23. Voshaar ORC, Banerjee S, Horan M, Baldwin R, Pendleton N, Proctor R, et al. Fear of falling more important than pain and depression for functional recovery after surgery for hip fracture in older people. Psychol Med. 2006;36(11):1635-45.

24. Bhala RP, O’Donnell J, Thoppil E. Ptophobia. Phobic fear of falling and its clinical management. Phys Ther. 1982;62(2):187-90.

\section{Wallace Hetmanek dos Santos}

Universidade Aberta da Terceira Idade. Universidade do Estado do Rio de Janeiro (UnATI/UERJ). Rio de Janeiro, RJ, Brasil.

\section{Karen O. Santos}

Instituto de Psicologia. Universidade Estadual do Rio de Janeiro. Rio de Janeiro, RJ, Brasil 